Cita bibliográfica: De Jesús Contreras, D. y Medina, F. X. (2021). El papel de las empresas trasnacionales en la promoción y desarrollo del enoturismo. Estudios de caso etnográficos en México y España. Investigaciones Turísticas (22), pp. 304-326. https://doi.org/10.14198/INTURI2021.22.13

\title{
El papel de las empresas trasnacionales en la promoción y desarrollo del enoturismo. Estudios de caso etnográficos en México y España
}

\section{The role of transnational companies in promoting and developing wine tourism. Ethnographic case studies in Mexico and Spain}

Daniel De Jesús Contreras (iD, Universidad Autónoma del Estado de México, México djcontreras66@yahoo.com

\author{
F. Xavier Medina (iD, Universitat Oberta de Catalunya, España \\ fxmedina@uoc.edu
}

\section{RESUMEN}

El objetivo del presente artículo consiste en analizar el desarrollo del enoturismo en dos territorios rurales de América y Europa a partir del estudio de una empresa trasnacional líder en la producción de vinos espumosos y en la venta de experiencias turísticas. Siguiendo un planteamiento metodológico cualitativo que retoma como métodos al estudio de caso y a la etnografía multisituada, se analizaron las prácticas de la empresa para su posicionamiento como referente de la producción vitivinícola y del enoturismo en los territorios estudiados. Los resultados muestran cómo esta empresa reproduce un modelo de negocio global a través de alianzas estratégicas y mediante la movilización de recursos económicos, políticos y sociales, consolidando una marca-territorio que emplea para promocionar sus productos y servicios. Se concluye que la participación de esta empresa en el enoturismo sirve como estrategia de asentamiento, reafirmación de estatus y reconocimiento de liderazgo en el sector vitivinícola.

Palabras clave: globalización; enoturismo; empresas trasnacionales; etnografía multisituada; transformación territorial.

\section{ABSTRACT}

The aim of this article is to analyze the development of wine tourism in two rural territories in America and Europe, based on the study of a leading transnational company in the field of sparkling wines, and also on the sale of tourist experiences. Following a qualitative methodological approach using a case study and multi-site ethnography, the practices deployed by the company were analyzed in terms of its positioning as a benchmark for wine production and 
wine tourism. The results show how this company implements a global business scale model, through strategic alliances and by mobilizing economic, political and social resources, consolidating a brand-territory used to promote its products and services. It may be concluded that the participation of this company in wine tourism serves as a settlement strategy, a reaffirmation of status and recognition of leadership in the wine sector.

Keywords: globalization; wine tourism; transnational companies; multi-site ethnography; territorial transformation.

\section{INTRODUCCIÓN: GLOBALIZACIÓN, TRASNACIONALISMO Y ENOTURISMO}

La globalización, inicialmente entendida como un proceso de integración de las sociedades y economías nacionales/regionales en la red global de comercio, comunicación y transportes, actualmente se comprende como un fenómeno social que trasciende la internacionalización de los mercados; define los procesos de intensificación de las relaciones sociales internacionales que interconectan lugares distantes, provocando que ciertos eventos mundiales tengan afectaciones locales, y viceversa (Dwyer, 2015). Refiere un fenómeno multiescalar que tiende continuamente a la homogeneización de diversos aspectos de la vida social; sin embargo, al mismo tiempo, crea espacios transnacionales para la revalorización de las culturas locales (Beck, 1998), permitiendo la heterogeneidad y la diferencia como reacción a la integración de las sociedades, los estados-nación y las economías nacionales (Appadurai, 2001).

El turismo es un ejemplo de este contrasentido: es reflejo de una industria globalizada que incorpora procesos del capitalismo y la modernidad, como la tercerización de los sistemas económicos, el despliegue tecnológico de sistemas de comunicación-información y la hegemonía de corporaciones trasnacionales (Marín y García, 2012, p. 1). Por otro lado, sin embargo, constituye un mercado que está valorando la singularidad de las experiencias; la participación directa de las comunidades locales a través de mecanismos de gestión y concertación; el respeto del entorno natural y sociocultural; así como la revalorización de las expresiones culturales autóctonas (Ávila, 2015). Esta nueva faceta del turismo retoma la dimensión territorial del desarrollo como vector axial de su práctica. Las transformaciones que promueve y los nuevos patrones de acumulación que suscita, están ligados a los intercambios globales que se concretan en entornos localizados.

Los espacios rurales constituyen un escenario donde se desarrollan las dinámicas de la globalización mediante la práctica del turismo. La multifuncionalidad del territorio, tantas veces argumentada como estrategia de diversificación productiva, se convierte en un discurso que incorpora los territorios rurales a procesos de globalización (Jiménez, 2014). Según estos planteamientos, en los espacios rurales operan prácticas que revalorizan la singularidad de los elementos materiales e inmateriales a través de su mercantilización y posterior incorporación a flujos económicos globales, como el turismo (Guinjoan et al., 2016).

La presencia hegemónica de corporaciones trasnacionales que ostentan capital económico es una característica inherente al turismo global (Marín y García, 2012). Concentran los recursos para la operación de los servicios turísticos, y su injerencia en la transformación de 
los territorios se basa en la maximización de modelos de negocio sustentados en acumulación económica y control territorial a partir de alianzas estratégicas con gobiernos y/o empresarios locales. Suelen ser organizaciones con presencia internacional en diferentes países y asociadas a grupos corporativos asentados en lo más alto de la pirámide social y económica mundial.

El trasnacionalismo es un fenómeno que surge de una nación y, desde ahí, se extiende. A diferencia de los procesos globales, que afectan a personas de todo el mundo y se encuentran descentralizados, los procesos trasnacionales están anclados en territorios nacionales concretos; ambos hacen referencia a fenómenos transfronterizos (Rivero, 2017). El turismo es un fenómeno global que incorpora procesos trasnacionales mediante la expansión de empresas multinacionales y sus filiales a diferentes regiones geográficas, siempre acompañadas de mayor poder financiero, tecnológico y organizacional (Romero y Vera, 2014). El asentamiento de estas organizaciones ocurre con mayor frecuencia en destinos cosmopolitas o de sol y playa, pero en los espacios rurales también es posible observar su papel en la transformación territorial y el desarrollo socioeconómico.

En el enoturismo, por ejemplo, una parte significativa de la experiencia tiene lugar en las bodegas vinícolas, algunas de las cuales son grandes empresas asociadas a grupos corporativos trasnacionales, que suelen contar con los recursos económicos, técnicos, humanos y de infraestructura para implementar actividades de ocio y para influir determinantemente en el desarrollo de las regiones vitivinícolas. Siguiendo los planteamientos de Lacarrieu (2009), para que la trasnacionalización de las diversas formas de turismo (incluido el enoturismo) pueda concretarse, es necesaria la hiperlocalización del patrimonio, de los recursos, de la información y del territorio mismo. Este vendría a ser el marco de actuación donde se instauran las relaciones locales-trasnacionales, a través de las cuales ciertos espacios, sociedades y sus relaciones se insertan a procesos globales.

Este trabajo analiza el papel de una empresa trasnacional del mundo del vino en la promoción y desarrollo del enoturismo en dos territorios, uno de América y otro de Europa. A través de este caso buscamos demostrar cómo los espacios rurales se insertan en procesos de globalización mediante prácticas trasnacionales asociadas a la reproducción de modelos de negocio, con especial énfasis en el enoturismo. A continuación de este apartado introductorio, se analiza la participación de las grandes empresas del mundo del vino en la promoción del enoturismo. Seguidamente, se presenta la metodología empleada, destacando el uso del estudio de caso y la etnografía multisituada. Posteriormente, se estudia el caso de la compañía Henkell-Freixenet en relación con su configuración como trasnacional vinícola, aspecto que sirve para contextualizar la especificidad del modelo de enoturismo desarrollado por la empresa en los territorios estudiados y ejemplificar la construcción de relaciones locales-globales. Finalmente, se presentan las conclusiones de la investigación.

\subsection{Enoturismo y grandes empresas vinícolas}

El enoturismo es una actividad ligada al disfrute de las técnicas agrícolas del cultivo de uva y de los procesos de elaboración de los vinos (Serrano, 2011), pero no solamente: también implica visitas a bodegas y asistencia a eventos (ferias, festivales, demostraciones) 
cuya motivación es la apreciación sensorial y el conocimiento de las culturas vitivinícolas (Brochado et al., 2019).

Para algunos autores se trata de una forma de turismo cultural porque el vino es en sentido amplio- un alimento y forma parte de la cultura alimentaria de un determinado lugar; es decir, de aquello que las personas comen y beben (Medina, 2015). En este sentido y desde un punto de vista antropológico, es un bien que refleja conocimientos y expresiones sociales transmitidas a través de generaciones, formando parte de la identidad y el patrimonio cultural (Wang, 2020), y su interés desde la perspectiva turística estaría relacionado con la cultura material e inmaterial. Sin embargo, no hay que olvidar que está fuertemente asociado al sector agrario y que, por lo tanto, una parte significativa del proceso de producción se asienta en territorios predominantemente rurales. Si una etapa de la experiencia consiste en visitar los paisajes conformados por viñedos, bodegas y algunos atractivos complementarios, claramente podríamos situarlo también como una forma de turismo rural o de turismo en espacios rurales (Holmes, 2017; Visentin y Vallerani, 2018).

Independientemente de su origen semántico y evolución conceptual, actualmente el enoturismo ha configurado una tipología propia (Mitchell y Hall, 2006; Peris-Ortiz et al., 2016) que incorpora elementos patrimoniales y se desarrolla fundamentalmente en los espacios rurales. Ofrece oportunidades de crecimiento para los territorios vitivinícolas a través de la valorización de elementos paisajísticos, históricos, culturales o ambientales que generan atracción a través de productos turísticos planificados (Ferreira y Hunter, 2017). Su desarrollo comienza como una acción aislada de algunas bodegas con la capacidad de infraestructura y recursos humanos para ofertar servicios de ocio, y se consolida mediante la creación de rutas y la formación de organismos de promoción (Favero y Rotta, 2007).

De acuerdo con Medina (2011), las rutas alimentarias -entre las que quedan incluidas las rutas del vino- son (deberían ser) desarrolladas de forma conjunta entre el sector público y privado; la financiación suele ser mixta, pero la mayor parte del capital, principalmente estructural, es aportado por las administraciones públicas. Sin embargo, existen casos en los que esta condición no se cumple y son los empresarios privados quienes encabezan las iniciativas turísticas. En Jalisco (México) se promueve la Ruta del Tequila, pero es ofertada y controlada únicamente por una empresa, mientras que los productores artesanales no forman parte del proyecto (Hernández, 2009) y la participación de las administraciones públicas se reduce a acciones de promoción y facilitación de infraestructuras. Otro ejemplo es el de las rutas del cacao en el sureste mexicano: una empresa trasnacional administra el museo, que es el principal atractivo de la ruta (Suremain, 2017).

En el caso del enoturismo nos encontramos ante dos realidades contrastantes; por una parte, existen rutas del vino formadas principalmente por pequeñas bodegas de tradición familiar-local y con producciones en pequeña escala (Fernández, 2012), mientras que otros proyectos son liderados por grandes bodegas que registran visitas masivas (Alberdi, 2018). En España, Freixenet y Codorniu en la Denominación de Origen (D.O) Penedès y González-Byass en la D.O. Jerez, llegaban a concentrar hace ya casi una década, más de 100 mil visitantes anuales, lo que ha representado desde entonces una importante fuente de diversificación de las ventas (Millán, 2012). Como muestra de su importancia en la industria vitivinícola y en 
el sector enoturístico, Tío Pepe (perteneciente a la firma González-Byass) es la bodega más visitada de Europa con 220 mil turistas anuales (Calleja, 2018), mientras que Freixenet se ha posicionado como la multinacional española ${ }^{1}$ más importante en el mundo del vino y el cava. En este marco, y tal como fuera observado por Medina (2015) en la región vitivinícola de TokajHegyalja (Hungría), el desarrollo de diferentes estrategias depende de las dimensiones y de las posibilidades de las distintas empresas. Las grandes apuestan por las visitas turísticas para promocionar sus productos y marcas, mientras que las pequeñas para diversificar las ventas y complementar los ingresos económicos.

Tabla 1. Empresas vinícolas con fuerte presencia en el enoturismo en Iberoamérica

\begin{tabular}{|c|c|c|c|}
\hline Autor (es) & País & Región de estudio & Empresas dominantes \\
\hline Zamora y Barril (2007) & Chile & Valles Centrales & Concha y Toro, Viña San Pedro \\
\hline Fávero y Rotta (2007) & Brasil & Valle de los Viñedos & Bacardí, Chandon de Brasil \\
\cline { 1 - 2 } Medina y Tresserras (2008) & \multirow{2}{*}{ España } & D.O. Penedés & Codorniu, Torres, Freixenet \\
\cline { 1 - 2 } \cline { 3 - 4 } Millán (2012) & & D.O. Jerez & González-Byass (Tío Pepe) \\
\cline { 1 - 1 } Thomé et al. (2015) & México & Querétaro & Freixenet, La Redonda \\
\hline
\end{tabular}

Elaboración propia.

Los trabajos aludidos en la Tabla 1 permiten observar algunos puntos nodales con respecto a la participación de las grandes empresas en el enoturismo: cuentan con sólida presencia nacional e internacional, llegando a posicionarse en ciertos casos como multinacionales del sector vitivinícola; realizan inversiones constantes en investigación y desarrollo de tecnologías para innovar procesos productivos; cuentan con productos y marcas reconocidas en el mercado del vino, lo que les confiere estatus y prestigio social; invierten fuertes cantidades en infraestructura y promoción de servicios turísticos y concentran flujos masivos de visitantes. Sin embargo, cabe precisar que dichos trabajos no fueron formulados para analizar específicamente el papel de las empresas, sino que su participación se comprende dentro de un contexto más amplio relacionado con el desarrollo socioeconómico de los territorios vitivinícolas.

En este sentido, y con excepción de estos pocos casos, hasta el momento no se ha documentado en el ámbito iberoamericano/latinoamericano el papel de las grandes bodegas, inversores extranjeros o empresas multinacionales en la promoción y desarrollo del enoturismo -más allá de los aspectos económicos implicados- quedando pendiente indagar cómo están reconfigurando los territorios enológicos donde se establecen a partir de la incorporación de actividades turísticas vinculadas al vino (y a la gastronomía). Lo anterior llevó a plantearnos algunas preguntas: ¿Por qué las grandes empresas vinícolas incursionan en el enoturismo? ¿Qué implicaciones territoriales y socioeconómicas están generando en los lugares donde se asientan? ¿Cómo, a partir de su presencia en más de un territorio, se construyen relaciones de tipo trasnacional?

1. Hay que destacar que Freixenet fue, sin embargo, vendida por su accionariado principalmente familiar, al grupo alemán Henkell en 2018. 


\section{METODOLOGÍA}

Este artículo analiza el caso de la trasnacional Henkell-Freixenet en relación con su incursión en el sector turístico. La hipótesis planteada consideraba que, si el éxito internacional de la empresa se ha fundamentado en la creación de economías de escala a través de la reproducción de un modelo productivo, la misma situación se observaría en el caso del enoturismo y, en un sentido más amplio, ello permitiría constatar la construcción de una relación local-trasnacional (Lacarrieu, 2009) mediante la cual la región vitivinícola se inserta en el proceso de globalización.

Lo anterior requirió de un planteamiento metodológico cualitativo para profundizar en las prácticas y discursos de la empresa. Se seleccionó, por un lado, el método de estudio de caso (Stake, 2000), y por el otro, la etnografía multisituada o multilocal (Marcus, 2001). El primero para la fase de diseño de la investigación, mientras que el segundo en el trabajo de campo. El estudio de caso se plantea como abordaje metodológico cuando se está frente a fenómenos nuevos o de los que se ha estudiado poco (cf. Tight, 2010); en este caso, aunque el enoturismo ha sido ampliamente estudiado en diversos contextos geográficos y socioculturales, poco se ha investigado sobre el papel que las grandes bodegas y empresas trasnacionales desempeñan en la promoción y desarrollo del enoturismo, salvo algunas excepciones (cf. Tabla 1) que, igualmente, muestran análisis parciales.

Los casos paradigmáticos se caracterizan porque ejemplifican los atributos o rasgos de un grupo, una población o una sociedad, presentándose como prototipos de un determinado universo de casos (Giménez y Heau, 2014). El diseño de la investigación se planteó mediante la segunda categoría propuesta por Yin (2009): estudio de un solo caso con unidades de análisis contenidas dentro del mismo. El caso seleccionado fue Henkell-Freixenet, entendida como multinacional vitivinícola, mientras que las unidades de análisis fueron las bodegas localizadas en la comarca del Penedès (Cataluña) y en Ezequiel Montes (Querétaro).

Sobre los criterios para delimitar el caso se consideró lo siguiente: a) es una empresa líder en el mercado internacional del vino; b) es la bodega más visitada dentro de un conjunto relativamente amplio en ambos territorios; c) ha desarrollado un modelo de visitas sobre la base de las especificidades productivas (vinos espumosos) y territoriales y c) es un referente de la producción vitivinícola y del desarrollo del enoturismo en las dos regiones.

Para el trabajo de campo, la elección de la etnografía multisituada se debió a que, al contrario que la etnografía tradicional, focalizada en la particularidad de una comunidad, grupo social o contexto, ésta propone superar las fronteras espaciales para seguir al objeto de estudio en una "pluralidad de lugares", involucrando más de un territorio donde se lleva a cabo el proceso de investigación (Perret, 2011). Se enfoca en una perspectiva trasnacional, pasando de un estudio de los sitios al análisis relacional entre estos; es decir, interesa comprender cómo se desarrolla el mismo fenómeno en otros contextos (Rivero, 2017). Este método se eligió porque buscábamos comprender los vínculos de la multinacional con los territorios donde ha desarrollado sus filiales y ha generado economías de escala, y ver cómo esto ejemplifica la inserción de territorios localizados en procesos globales más amplios a través del enoturismo, ya que, como sostiene Marcus (2001 p. 112), este método, al mismo tiempo que es una 
etnografía del contexto estudiado, es también una expresión etnográfica del sistema mundo, porque sitúa el análisis en un campo donde se tejen las conexiones entre ambos contextos (global y local). Así, más que realizar un ejercicio comparativo tradicional-como propone el estudio de caso- lo que interesa es indagar el tipo de relaciones y/o conexiones y no sólo la dualidad global-local. Para Marcus (2001 p. 116) la etnografía multilocal permite comprender cómo "lo global se colapsa en, y es vuelto parte integral de, situaciones locales paralelas y vinculadas entre sí, más que como algo monolítico o externo a ellas".

El trabajo de campo se desarrolló sobre el terreno para el caso mexicano en diversos períodos entre 2016 y 2018, con visitas discontinuas a lo largo de 2019. En el caso español, se realizó trabajo de campo intensivo durante tres meses, en 2017. Debe tenerse presente que una característica de la etnografía multisituada es la variabilidad en intensidad y amplitud de la información recabada (Marcus, 2001), por lo que este trabajo no es la excepción.

Los datos se obtuvieron a través de técnicas clásicas como observación participante y entrevistas en las unidades de análisis, atendiendo, en este último caso, a las especificidades impuestas desde el campo de la antropología de la alimentación (Medina, 2004). En cuanto a la primera, es una de las técnicas más útiles cuando se busca conocer las prácticas sociales en la cotidianidad (Jociles, 2018). La observación participante en ambas unidades de análisis posibilitó un registro detallado de los elementos puestos en valor para el enoturismo y nos proporcionó una aproximación más precisa sobre las prácticas y discursos movilizados.

Respecto a la segunda, la elección de informantes clave fue un recurso valioso para corroborar la información recabada durante las observaciones, pero también para ampliar el horizonte de conocimiento sobre las conexiones entre ambas unidades estudiadas. La elección de los informantes se realizó mediante un muestreo no probabilístico e intencional atendiendo a los siguientes criterios: a) conocer la estructura y funcionamiento de la empresa; b) expresar alguna relación (laboral, de negocios, formal o informal) pasada y/o presente; c) conocer el desarrollo del enoturismo en los territorios estudiados a partir de la injerencia de la multinacional y, finalmente, d) haberse movido entre ambas unidades de análisis. Sobre esto último, solamente tres informantes cumplieron con el criterio. Los dos restantes demostraron conocimiento de las conexiones entre ambas unidades por lo que en forma de narrativas pudieron comprender por sus vínculos con la empresa, así como por información de redes sociales y medios digitales de comunicación. Las entrevistas proporcionaron una visión más amplia y desde diferentes ángulos (Medina, 2004) para comprender cómo se configuran las relaciones trasnacionales.

Aunque la presencia física del investigador en los contextos de estudio es deseable, esta no siempre es una limitante, pudiéndose seguir al objeto de estudio a través de las narrativas y discursos (Rivero, 2017). Así, este trabajo incorpora el análisis de fuentes secundarias como textos académicos, notas de prensa, redes sociales y material audiovisual.

El análisis de los datos se realizó mediante el procedimiento inductivo propuesto por Shaw (1999): primero se transcribieron las entrevistas, notas de campo e información relevante de las fuentes secundarias; posteriormente se realizó una comparación constante de los temas que emergían (como la instauración de un modelo productivo y el desarrollo de 
un modelo particular de enoturismo); finalmente, se realizó un análisis más profundo contrastando dichos temas con los conceptos reportados en la literatura para establecer las conexiones trasnacionales entre las unidades analizadas.

\section{RESULTADOS}

\subsection{Consolidación de Freixenet como trasnacional vinícola}

Freixenet es una de las multinacionales más conocidas en el mundo del vino, cuya matriz se localiza en la localidad de Sant Sadurní d'Anoia, en la comarca catalana del Alt Penedès, en la provincia de Barcelona. Desde su fundación en 1911 se ha especializado en la elaboración y comercialización de Cava², vino espumoso que sigue el tradicional método champenoise.

El proceso de internacionalización y su posicionamiento como transnacional del vino se basa en exportaciones, alianzas comerciales y creación de filiales (Pedrero, 2015). La expansión de la empresa tuvo un carácter inicialmente regional: la introducción del cava se realizó primero en la ciudad de Barcelona y después en toda Cataluña. Posteriormente, los vinos se comercializaron en las regiones de Aragón y Valencia, para después introducirse en Madrid y el resto del mercado español (Blanco, 2016).

Además de exportaciones y alianzas comerciales en diferentes países, la política de expansión de la empresa considera la creación de economías de escala mediante el establecimiento de filiales que producen vino en el mismo país. Los beneficios se traducen en la eliminación de intermediarios, la producción de grandes volúmenes, la adecuación de los productos a los mercados locales y la consolidación de una logística eficiente (Pedrero, 2015).

La internacionalización de Freixenet tuvo un éxito comercial y económico sin precedentes: entre 1976 y 1985 pasó de exportar 300.000 botellas a 20.000.000, lo que representaba el $72 \%$ de las exportaciones; es decir, se consolidaba como una empresa multinacional y líder en la exportación de cava. En paralelo a su proyección internacional, entraba en nuevos mercados en España con la elaboración de vinos tranquilos en importantes D.O. como Ribera del Duero, Rioja, Priorat, Montsant o Rías Baixas (Blanco, 2016).

La relación calidad-precio fue decisiva en la conquista del mercado nacional y en la proyección internacional porque supuso la introducción de innovaciones tecnológicas en el proceso tradicional: fermentación del mosto en depósitos refrigerados y sustitución de pupitres empleados en el removido manual de botellas por contenedores de decantación (Pedrero, 2015). Otro elemento para considerar es el posicionamiento de marca mediante productos emblema, innovaciones constantes e inversiones en marketing y publicidad.

2. Los vinos elaborados y comercializados bajo la Denominación de Origen Cava proceden únicamente de Cataluña y de algunas otras áreas específicas en España. En los demás países se emplea el término "vino espumoso", "espumante" o similares. 
Tabla 2. Bodegas y marcas de Grupo Freixenet

\begin{tabular}{|c|c|c|}
\hline País & Región & Bodega/Marca \\
\hline \multirow{15}{*}{ España } & \multirow{5}{*}{ Comarca del Penedés, Cataluña } & Segura Viudas \\
\hline & & René Barbier \\
\hline & & Castellblanc \\
\hline & & Canals \& Nubiola \\
\hline & & Conde de Caralt \\
\hline & \multirow{2}{*}{ Comarca del Priorat, Cataluña } & Morlanda \\
\hline & & Fra Guerau \\
\hline & Comarca del Empordà, Cataluña & Cop de Vent \\
\hline & Rias Baixas, Galicia & Vionta \\
\hline & Ribera del Duero, Castilla y León & Valdubón \\
\hline & \multirow{2}{*}{ Rueda, Castilla y León } & Fray Germán \\
\hline & & Etcétera \\
\hline & Rioja Alavesa, País Vasco & Solar Viejo \\
\hline & Comunidad de Madrid & Torresano \\
\hline & Alicante, Comunidad valenciana & Nauta \\
\hline \multirow{2}{*}{ Francia } & Reims, Marne & Henri Abelé \\
\hline & Gironde Sur Dropt & Yvon Mau \\
\hline Estados Unidos & Sonoma, California & Gloria Ferrer \\
\hline México & Ezequiel Montes, Querétaro & Sala Vivé \\
\hline Australia & Melbourne & Wingara Wine Group \\
\hline Argentina & Valle de Uco, Tupungato & Viento Sur \\
\hline Chile & Valle de Curicó & Terra Nova \\
\hline Japón & & Oroya \\
\hline
\end{tabular}

Fuente: $\underline{\text { http://www.grupofreixenet.com/ }}$

En el primer caso, la creación de dos productos emblemáticos: Carta Nevada en 1941 (relanzado al mercado en 1967) y Cordón Negro en 1974, generaron una identidad de marca y un modelo de los estándares de calidad de la empresa, debido a las innovaciones realizadas en la presentación del producto: en lugar de utilizar la tradicional botella verde, la empresa apostó por botellas esmeriladas, blanca para Carta Nevada y negra para Cordón Negro. En la década de 1980, Cordón Negro era su producto más vendido en Estados Unidos; hacia 2014 se encontraba distribuido en 142 países a través de las distintas filiales (Blanco, 2016).

La otra parte del éxito se relaciona con las agresivas inversiones en campañas publicitarias para promocionar los productos y, consecuentemente, la marca de la empresa. Los comerciales del Cava Carta Nevada en televisión en Navidad, por ejemplo, se convirtieron en un ícono de la estrategia publicitaria y le aseguraría un importante margen de ventas. La estrategia consistía en promocionar de forma original los productos en medios masivos de 
comunicación para abarcar diferentes segmentos de mercado, para lo cual recurrieron a la contratación de personajes populares ligados al cine o la televisión, cantantes y deportistas, entre otros (Furió, 2012).

Las claves del éxito internacional de Freixenet se pueden resumir en: a) creación de economías de escala y alianzas comerciales; b) inversiones constantes en innovación y desarrollo de productos; c) mercadeo basado en la imagen de la marca a través de la publicidad; d) relación calidad-precio de los productos ofertados y e) expansión de la compañía a diferentes países y distintos mercados (Blanco, 2016). Actualmente, sus productos se encuentran en 120 países, cuenta con 20 filiales y más de 20 bodegas y marcas distribuidas internacionalmente (tabla 2) y factura ventas que superan los 740 millones de dólares (2016). ${ }^{3}$

\subsection{La apuesta de Freixenet por el turismo en la comarca del Penedès}

La apertura de Freixenet al enoturismo se inició en la década de 1970 y coincide con los planes de internacionalización puestos en marcha años atrás. Corresponde a la intención de diversificar las ventas mediante el consumo in situ y en diferentes épocas del año, ya que tradicionalmente el cava ha estado ligado a celebraciones. Freixenet es una de las bodegas pioneras -junto con Torres y Codorniu- del enoturismo en el Penedès, introduciendo en España un modelo de enoturismo consistente en la recepción de visitantes para hacer recorridos por las instalaciones, conocer los procesos productivos y degustar los vinos; asimismo, incorporaron el servicio de tienda para la venta de souvenirs (Molleví y Fusté, 2016), con los cuales se busca generar identidad de marca, y posicionarla como la multinacional líder en la elaboración de cava, pero también en la venta de experiencias turísticas a nivel comarcal, nacional y mundial.

Desde luego y tal como apuntan Molleví y Fusté (2016), este modelo está muy influenciado por la incursión de la empresa en el mercado norteamericano, de donde adquirieron experiencia que luego implementaron en las demás filiales abiertas al público. En cierto sentido, la trasnacionalización hizo posible la adopción y estandarización de un modelo enoturístico que se comercializa bajo la misma marca que los vinos. Sin embargo, el modelo de visitas implementado era rígido y culminaba con la degustación de productos de la marca; las necesidades de diversificación y complejidad de la oferta, dictadas por el dinamismo del mercado turístico, obligaron a reconfigurar el modelo y apostar por una estrategia que combina tradición, innovación, calidad y entretenimiento.

En este marco, se reconoce la existencia de tres modelos diferenciados de enoturismo, dependiendo del contexto territorial donde se localizan las filiales:

Freixenet tiene tres [modelos], en Estados Unidos tiene uno, aquí [España] tiene otro y tiene el que tiene en México, pero aquí todo el mundo tiene el mismo; es

3. Véase https://www.statista.com/statistics/232865/leading-20-most-valuable-alcohol-spirits-brands-worldwide/ 
decir, todo España tiene el mismo, que es el de contar la tradición y contar cómo se hace el vino y desde cuantos años llevamos haciendo vinos [...] El modelo nuestro de facturación en enoturismo, de elite, VIP, de calidad, es el de Sonoma [...] la gente llega porque quiere llegar. [En México] es un híbrido, han sabido aunar la tradición que tienen porque si que es verdad que históricamente la tienen, porque al final entraron las viñas a todo lo que es América a través de México, pero con un modelo mucho más de entretenimiento [...] entretenimiento puro y duro (Informante 2, entrevista personal, 2017).

Esta información fue corroborada durante las visitas a la bodega: un momento crucial de la experiencia es el recorrido por las cavas centenarias construidas a 20 metros de profundidad, que incluye también la parte moderna, donde se localizan las nuevas tecnologías de producción. Durante las explicaciones, las guías enfatizan la historia de la bodega, reafirmando su papel en el desarrollo del territorio y posicionándose como una compañía familiar líder en la tradición de elaborar cava, de la cual constan como testigos las cavas labradas en rocas subterráneas, herramientas antiguas y botellas vetustas.

La insistencia en el componente histórico y cultural se ve asimismo reflejada en experiencias turísticas enogastronómicas, que aumentan la dimensión de entretenimiento del vino a través de maridajes con la gastronomía local: cocina catalana y productos emblemáticos de la comarca. Es interesante observar cómo este tipo de experiencias promocionan el territorio por la vía sensorial, pero desde la perspectiva de la multinacional; es decir, instaura su propio concepto de enoturismo, fundamentado en las asociaciones posibles entre viticultura y gastronomía, cuyo soporte es el espacio productivo y rural en tanto que ejes de consumo turístico (Guinjoan et al., 2016).

Es importante mencionar que Freixenet se asienta en la región enológica más importante de Cataluña y en una de las más sobresalientes de España (Medina y Tresserras, 2008), que para 2018 registró 441.467 visitantes, convirtiéndose en la segunda región vitivinícola más visitada, sólo después de la Ruta del Vino del Marco de Jerez (ACEVIN, 2019). De acuerdo con lo relatado por nuestros informantes, las tres bodegas pioneras captan el $90 \%$ de las visitas y tan solo Freixenet llega a contabilizar más de 100.000 visitantes anuales, con un gasto medio de 3.4 millones de euros. ${ }^{4}$ El turismo es, pues, una importante estrategia comercial que permite incursionar en un mercado en constante crecimiento y que, asimismo, genera fidelidad hacia los productos de la marca.

4. El cálculo se realizó considerando los datos reportados por ACEVIN (2019), que estimó para 2018 un gasto promedio de 34.88 euros: 14.99 euros considerando el precio medio por visita estándar establecido por las bodegas del Penedés y 19.89 euros de gasto medio en tienda por concepto de vinos y souvenirs. 
Figura 1. Cavas Freixenet, en Sant Sadurní d’Anoia
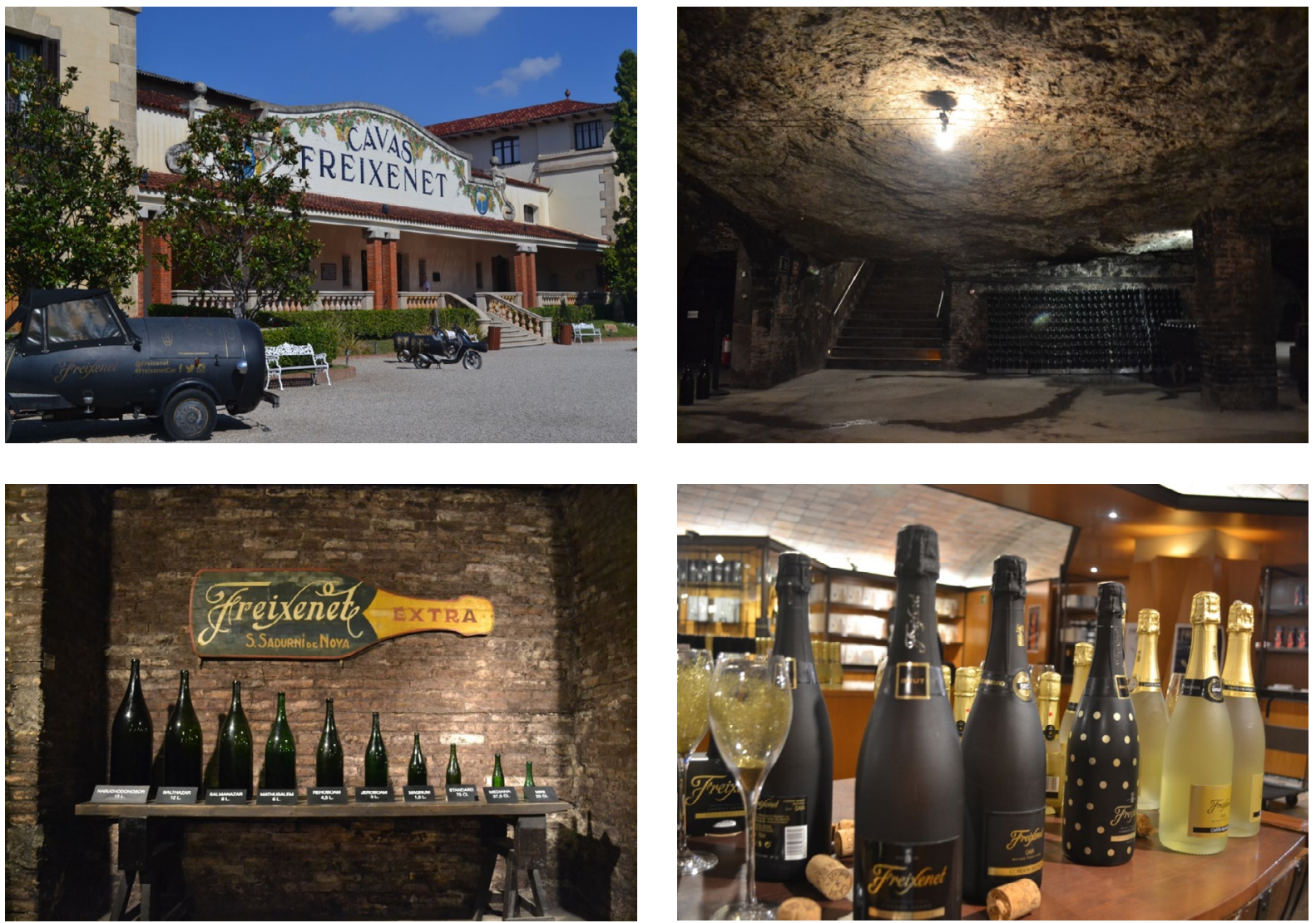

Fuente: D. De Jesús (2017).

Pese a situarse dentro del territorio de la D.O Penedès, los vinos espumosos elaborados pertenecen a la D.O Cava, específica para este tipo de vinos. No obstante, al contar con otras bodegas del mismo grupo en la primera, la empresa también elabora y comercializa vinos tranquilos bajo la D.O Penedès. Estas bodegas cumplen una función estratégica de complementariedad de la oferta en la matriz, al mostrar aspectos que en ésta no se evidencian, como por ejemplo, la relación de los productos con el territorio a través de los viñedos y el paisaje. La delimitación territorial es muy importante en el marco del turismo, ya que el territorio en general se promociona mediante la marca Enoturisme Penedès (Thomé et al., 2015), que incluye las bodegas de la D.O Cava; más allá de la promoción de un producto específico, como ocurría anteriormente con la Ruta del Vi i el Cava (Ruta del vino y del cava), se promociona un territorio.

Una de las principales fortalezas del territorio y de Freixenet en particular, es la especialización productiva ${ }^{5}$ en torno al cava, y ello es ya en sí mismo un elemento de diferenciación respecto a la oferta de enoturismo de otras regiones españolas. Así, el liderazgo de Freixenet se explica en parte por el marco territorial que la contextualiza: el territorio funciona como

5. En adelante, cuando se haga mención de este concepto nos referimos a la idea original desarrollada por los autores para el caso específico del vino. 
soporte de las actividades ofertadas. Un punto interesante a destacar es la creación de alianzas comerciales con el Centro de Interpretación del Cava (CIC Fassina), que consiste en visitas combinadas al centro y luego a la bodega. Asimismo, cuentan con un paquete específico denominado Freixetren, que incluye el viaje en tren desde Barcelona hasta Sant Sadurní d'Anoia, la visita guiada, más la degustación de dos cavas. Lo que se puede apreciar con las estrategias comerciales establecidas a nivel de comarca es un claro ejemplo de una de las claves del éxito de Freixenet a nivel internacional: alianzas comerciales con socios estratégicos.

Al margen de los irrisorios beneficios económicos derivados del enoturismo en la bodega matriz, en comparación con los volúmenes de ventas de cava y vino a nivel mundial, los motivos reales de su incursión en esta actividad se relacionan con la reafirmación de la marca en el territorio y en el mercado español, como bodega líder en la producción y comercialización de cava y como una de las más visitadas y con mayor venta de experiencias enogastronómicas. Así lo confirmó nuestra informante:

El modelo de política enoturística viene muy apto del posicionamiento de la marca en el mercado de producto ¿ंvale? Y te digo, nosotros somos conocidos en el mundo por fabricar 100 millones de botellas, 80 de ellos de Carta Nevada y 20 de Cordón Negro y vender en 150 países: volumen y a precio [...] si tu quieres volumen, quieres volumen. [El enoturismo] funciona como una política de posicionamiento de marca a nivel internacional (Informante 3, entrevista personal, 2017).

Nos encontramos frente a una empresa que implementa un modelo enoturístico masificado -con precios asequibles y dirigido a diferentes segmentos de mercado- pero eficaz para el posicionamiento de la marca. Esto ha sido y es posible gracias a que la multinacional cuenta con un aparato sólido de promoción y marketing, una marca consolidada y abundantes recursos económicos, sociales, culturales, humanos y físicos. Así, y aunque forma parte de la promoción de un territorio, su inclusión en el consorcio Penedès Turisme es más bien nominal, ya que los elementos descritos con anterioridad le permiten actuar de manera relativamente independiente ante las iniciativas colectivas para promocionar el territorio en su conjunto. Sin embargo, indirectamente genera un efecto positivo principalmente sobre el sector de la restauración debido a la promoción activa que realiza sobre la cocina catalana a través de sus productos turísticos.

Por otra parte, de acuerdo con lo narrado por nuestros informantes, la empresa busca innovar el modelo enoturístico hasta ahora comercializado mediante la reducción del número de visitas -con una oferta de servicios de mayor calidad y exclusividad para un segmento de alto poder adquisitivo- $y$, de esa manera, posicionar productos de alta gama; además y en congruencia con su filosofía, se pretenden crear alianzas con las otras dos grandes bodegas del territorio -sus competidoras directas- para desarrollar una potente oferta que posicione al Penedès como el principal destino enoturístico de España, con lo que indirectamente se reforzaría su presencia y liderazgo en el sector vitivinícola.

\subsection{Resurgimiento de la región enológica mexicana de Querétaro a través de Freixenet: especialización productiva y enoturismo}

En consonancia con sus planes de internacionalización, Freixenet llegó a México en 1978 y se estableció en el municipio de Ezequiel Montes, Querétaro, donde construyó Sala 
Vivé, cuyas operaciones comenzaron en 1986. En el momento de su llegada, la región enológica queretana atravesaba por una severa crisis económica que dificultaba el desarrollo de la vitivinicultura (De Jesús et al., 2019). Sin embargo, las condiciones edafoclimáticas y la ubicación geográfica en torno a importantes polos económicos (cf. Thomé, 2019) hicieron que los directivos mantuvieran la decisión de continuar con los planes de expansión internacional y, sobre todo, impulsar la vitivinicultura en el centro del país.

La crisis se intensificó en la década de 1990 como consecuencia de la firma del Tratado de Libre Comercio de Norteamérica, que promovía la liberalización del mercado comercial y la apertura a las importaciones. Tras este acontecimiento, los problemas productivos en la región queretana se agudizaron y obligaron a la empresa a cerrar la bodega durante algunos años, sin garantías para solventar la producción (De Jesús et al., 2019). Sin embargo, esto no significó un fracaso: la multinacional contaba con los capitales financieros y físicos, respaldados además por su liderazgo internacional, para mantener la filial de México.

Figura 2. Finca Sala Vivé en Ezequiel Montes
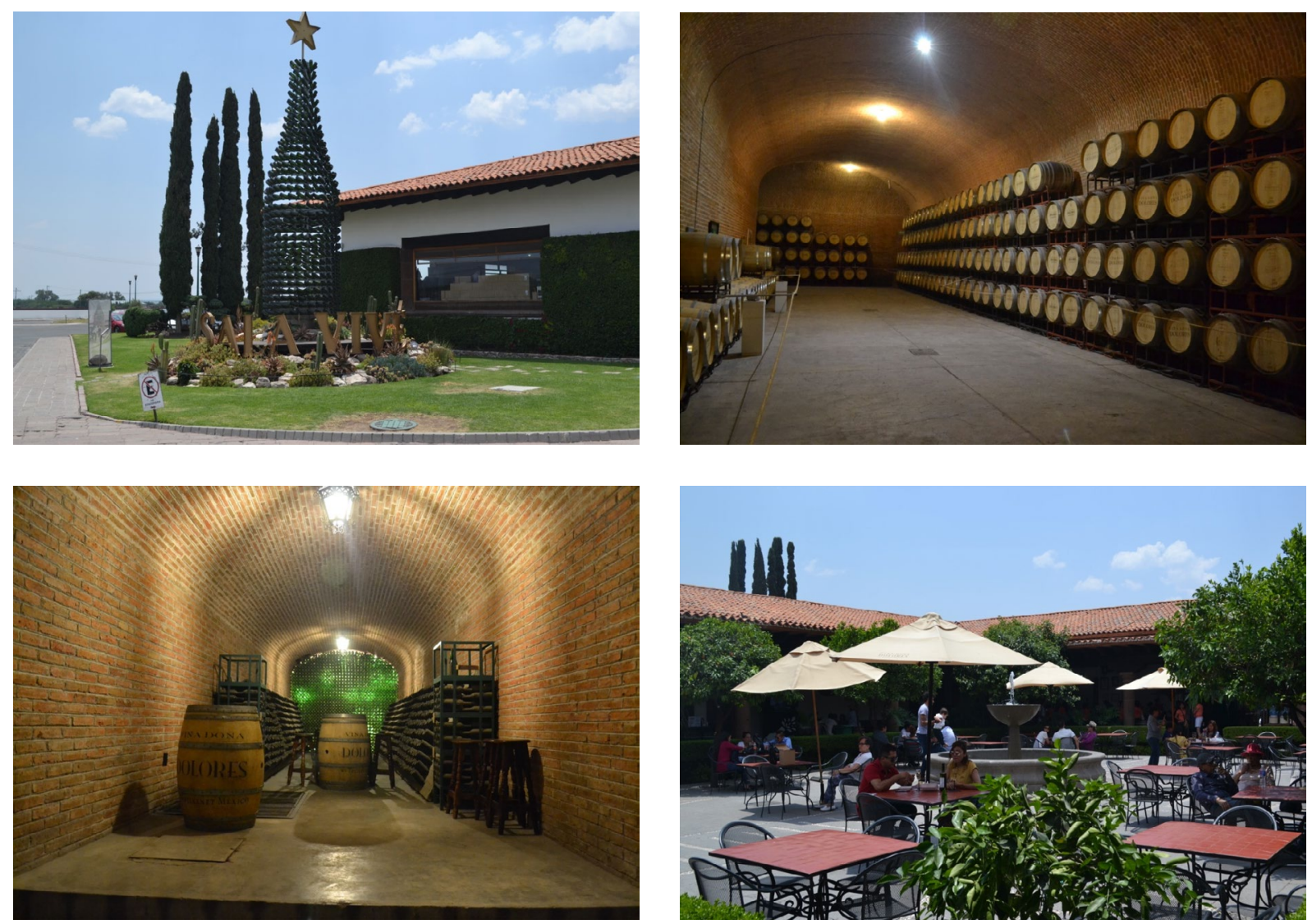

Fuente: D. De Jesús (2017-2018).

La visión estratégica de negocio, el liderazgo internacional y el estatus social fueron determinantes en la reactivación de las operaciones de la bodega y de la vitivinicultura en la región. La apuesta de Freixenet consistió en enviar enólogos desde la comarca del Penedès con instrucciones precisas para elaborar vinos espumosos. De acuerdo con lo relatado por nuestros 
informantes, a su llegada a territorio queretano las operaciones de la empresa se encontraban estancadas y buena parte de los ínfimos productos vendidos se exportaban desde Cataluña (alrededor de 40.000 botellas). La estrategia consistió en implementar la fórmula ensayada previamente en Cataluña: elaborar un producto estrella, en este caso un vino espumoso brut nature, y promocionarlo a través de las visitas (De Jesús et al., 2019).

Esto significó una reconversión de la vitivinicultura que hasta antes de la crisis había consistido en la elaboración de destilados y aguardientes de uva. Las variedades de vid se sustituyeron por cepas idóneas para vinos espumosos, entre las que destaca especialmente la variedad $x a r e l \cdot l o$, típica del Penedès y con la que se elaboran algunos de los cavas catalanes más afamados. A partir de entonces, inició una etapa de la vitivinicultura queretana caracterizada por la elaboración de vinos de mesa y, muy destacadamente, de vinos espumosos (De Jesús et al., 2020). Esta creciente especialización ha posicionado la región como la principal productora de estos vinos en México, con cerca de tres millones de botellas anuales; números que a su vez, están muy ligados a Freixenet y su tradición internacional en la elaboración de cava, concentrando cerca del 98\% de la producción de espumosos en México (Almanza, 2017a).

Freixenet ejerce como un nodo territorial alrededor del cual se ha gestado una reproducción a escala (Cf. Thomé et al., 2015) del territorio donde se asienta la matriz, y desde donde se ha construido la base de su éxito internacional en torno a un producto muy específico. La transformación territorial y especialmente, productiva, encabezada por la multinacional (De Jesús y Thomé, 2018), permite asimismo constatar cómo las tradiciones son sustraídas de su contexto y reintroducidas en otros territorios con las condiciones propicias para ello gracias a procesos trasnacionales; en cierto sentido se ha implantado una extensión territorial de la DO Cava por y para Freixenet. Un directivo de la bodega en México así parecía reconocerlo:

Yo digo, no se cuando va a suceder esto pero creo que [en] Querétaro las vinícolas acabarán vendiendo mas vinos espumoso que vino tinto [...] Creo que Querétaro se va a posicionar como la región de [...] los vinos espumosos de México [...] A nosotros nos ha ayudado mucho el enoturismo, recibimos muchas visitas aquí y la gente viene a tomar espumoso, puedes tomarte una paella, puedes tomarte quesos de la región e incluso tacos muy buenos y lo maridan con espumoso y estamos notando que eso nos ha ayudado mucho a que el consumidor conozca el espumoso (TomaVinoMexicano, 2019a).

La prensa escrita y algunos medios de comunicación han documentado esta tendencia en la región, bautizada como "la burbuja de México" (Almanza, 2017a). Es importante mencionar que la producción de vinos tipo Cava ha encontrado en el enoturismo un medio para su promoción y posicionamiento, en el cual y como elemento constitutivo de la experiencia, la gastronomía también forma parte, con una fuerte influencia mediterránea. La diversificación de los usos alimentarios del vino con la incorporación del enoturismo y la gastronomía (De Jesús et al., 2020) es la base sobre la que se construye la dimensión de entretenimiento que señalaba nuestra informante en Cataluña. Esta relación, que podríamos calificar de simbiótica, ha configurado la principal fortaleza y especificidad del modelo de visitas implementado en la bodega de México. Por ejemplo, el diseño de los recorridos guiados, eventos temáticos y gastronómicos retoma como eje principal la producción de vinos espumosos, siendo esta su 
principal ventaja comparativa respecto de otras bodegas concentradas en experiencias genéricas en torno a vinos tranquilos:

Es un fenómeno interesante lo que está sucediendo en Querétaro, este año cerraremos con 300.000 personas que han pasado por Finca Sala Vivé [...] estamos buscando si somos la vinícola más visitada del mundo, estamos viendo quien nos certifica esto [...] de México desde luego que si, de Europa también, porque la más visitada me parece que es Tío Pepe con 150 mil al año; ósea, nosotros los estamos doblando y realmente es un orgullo del trabajo que se ha hecho en los últimos 25 años en Finca Sala Vivé, que ha sido punta de lanza (TomaVinoMexicano, 2019a).

Debe destacarse la importancia cada vez más creciente del enoturismo para la bodega. Se estima que entre el $80 \%$ y el $90 \%$ de las botellas se venden en el contexto de las visitas, ya sea para consumo in situ o como souvenir turístico; además, la bodega es la más visitada de México y de Latinoamérica (AVQ, 2017). Algunos eventos temáticos de la empresa se han posicionado paulatinamente como insignias gastronómicas en la región: el Festival de la Paella, en su edición de 2017, acogió 12.000 visitantes y una derrama de 3.000 .000 de pesos (Almanza, 2017b), mientras que las Fiestas de la Vendimia han llegado a registrar hasta 15.000 visitantes (Almanza, 2017c).

En contraparte y coincidentemente con el modelo de visitas implementado en Cataluña, el de Querétaro se muestra masivo y muy poco especializado, a pesar de estar enfocado en los vinos espumosos y de contar con una amplia cartera de eventos y servicios. No obstante, no deja de ser un referente de lo que actualmente significa el enoturismo en la industria vitivinícola, y particularmente para Freixenet como compañía:

El éxito de México ha sido brutal [...] se sigue haciendo lo que hacía ella [refiriéndose a una persona clave, pionera del enoturismo en Querétaro] hace 80 9 años, pero masificado; es decir, no hay mucha nueva actividad ni tal, es un modelo que funciona solo, que tiene 260 mil personas y que tiene mucho gasto $y$ mucho consumo de botella en los eventos y al final es lo que quiere la compañía (Informante 3, entrevista personal, 2017).

La impresión de nuestra informante permite constatar que el éxito de la filial en México se explica en buena medida por la masificación de las visitas, lo cual le ha permitido llegar a un espectro de consumidores más amplio y, así, posicionar la marca. Dentro de la Ruta del Arte, Queso y Vino desempeña un liderazgo importante a partir de alianzas estratégicas con turoperadores locales, productores de queso y con la otra bodega más importante del territorio, La Redonda (Thomé et al. 2015; De Jesús et al., 2020). Este proyecto ha servido para reafirmar su liderazgo en el mercado mexicano del vino, pero también y más importante, afianzar su estatus como la empresa pionera del enoturismo y de la reactivación de la vitivinicultura en la región queretana (Thomé, 2019). Esto último es de particular relevancia puesto que el papel de la bodega en el desarrollo socioeconómico de la región ha sido determinante: gracias a la incorporación de actividades turísticas ligadas al vino se generó un mercado específico (turismo enogastronómico) del que se han visto directa e indirectamente beneficiados otros 
empresarios hoteleros, restauranteros y tour operadores; asimismo, para artesanos y productores de queso ha significado un importante canal de comercialización.

\subsection{La transnacionalización del territorio a través del enoturismo}

Uno de los principales cuestionamientos del caso analizado se refiere a las razones por las que las grandes empresas vinícolas incursionan en el mercado del turismo y la gastronomía. Particularizando el ejemplo de Freixenet, si esta empresa cuenta con una marca consolidada, si tiene fuerte presencia a nivel internacional y si los beneficios económicos derivados del comercio de vinos son amplísimos, ¿por qué se integra al turismo más allá de una aparente estrategia de marketing y promoción?

Una probable respuesta es que, muy en el tono de su liderazgo económico y empresarial, Freixenet no puede permitirse estar al margen de un segmento de mercado que le atañe directamente. La sinergia entre vino y turismo es cada vez más importante, llegando a establecer un mercado propio (Medina y Tresserras, 2007). En este sentido, la empresa catalana ve en el enoturismo un medio para mantener su prestigio e incluso para reafirmar su posición dentro del sector. Además de significar una estrategia comercial y económica, es una clara apuesta de reconocimiento social a través del posicionamiento de marca.

Lo anterior lleva a plantear que la internacionalización de Freixenet se realiza a través de la reproducción a escala (cf. Thomé et al., 2015) de un modelo de negocio sustentado en su visión empresarial, en su liderazgo como marca internacional de vinos espumosos y en su reputación y prestigio social dentro del mundo del vino, siendo estos los principales aspectos que denotan las conexiones local-trasnacional (Lacarrieu, 2009) entre las regiones analizadas. La instauración a nivel local de este modelo evidencia el avance del capitalismo hacia los espacios rurales y la incorporación de las regiones a la globalización (cf. Guinjoan et al., 2016) mediante la trasnacionalización (con la acentuada producción de espumosos y con la introducción del enoturismo, tal como veremos seguidamente).

En el marco del enoturismo y siempre bajo la lógica de su éxito internacional, Freixenet ha exportado un modelo de enoturismo basado en dos aspectos puntuales: especialización productiva y entretenimiento mediante experiencias enoturísticas y gastronómicas a partir de recorridos guiados, catas y maridajes con la cocina mediterránea. En México este es un aspecto muy evidente, y prueba de ello es la consolidación de un evento enogastronómico en torno a un plato emblemático (pero también ampliamente estereotipado) de la cocina española: la paella, maridada con el vino espumoso de la casa. Este ejemplo sirve también para ilustrar cómo en la región enológica queretana se está construyendo un modelo aspiracional que emula los destinos enogastronómicos europeos a partir de una producción constante y creciente de vinos, quesos o embutidos, entre otros productos, pero disociado de forma muy marcada de los recursos alimentarios locales (cf. De Jesús y Thomé, 2018), y evidencia la ya mencionada inserción del territorio a la globalización.

Por otra parte, la empresa ha implantado y consolidado en Querétaro la producción de vinos espumosos. Las implicaciones de este hecho no son menores: además del impacto económico y de la monopolización de un cierto saber hacer, se está construyendo una identidad 
territorial de toda la región desde y para una empresa. La especialización productiva, y ahora también territorial, desvela cómo en el plano local o regional se articulan las prácticas y narrativas que convierten a Freixenet en la trasnacional líder en la producción de cava. Así como a nivel global es innegable su reputación, prestigio y estatus social, también lo es a nivel local, siendo reconocida en la región queretana como la pionera en la reactivación de la vitivinicultura, situación que le confiere posicionamiento y centralidad tanto en el territorio como en el gremio de productores:

También hacemos un espumoso, método tradicional [...], eso es, no influencia pero si un poquito ánimo de Freixenet [...] nos ha animado a que tengamos todos nuestro espumoso, si ellos bien son el productor de espumoso más grande de México y son un representativo a nivel mundial como marca en espumosos o en cava, pues no está de más tener aquí en Querétaro ese vino (TomaVinoMexicano, 2019b).

La producción de vinos tipo Cava es su principal fortaleza y también su principal atractivo. Sin embargo, el modelo enoturístico de la empresa, y pese a los amplios beneficios en Querétaro, se muestra masificado y pasivo, coincidente con el modelo de desarrollo turístico mundial. No obstante, tiene un éxito inusitado que llega a representar el principal margen de venta, convirtiéndose en una importante estrategia de marketing y de diversificación de los ingresos económicos; en este sentido, al tiempo que los turistas visitan las instalaciones contribuyen al posicionamiento de la marca mediante el consumo in situ (De Jesús et al., 2020). Especialización productiva y enoturismo conforman un binomio que la empresa ha sabido explotar dentro de un mercado como el mexicano, ávido de experiencias pretendidamente auténticas y singulares.

Las alianzas comerciales en los territorios analizados son otra muestra de cómo se reproduce el modelo de negocio de Freixenet. En Querétaro, las alianzas con actores estratégicos conforman la estructura base de la Ruta del Arte, Queso y Vino. Más allá de los beneficios económicos, lo que resulta significativo es la hegemonía de la empresa: además de monopolizar la producción de vinos (tanto espumosos como tranquilos), también monopoliza las visitas (Thomé, 2019). En el caso de Cataluña sucede lo mismo, pero con connotaciones distintas: es una de las grandes vinícolas de la comarca y una de las que más visitantes recibe, pero su liderazgo y posicionamiento estratégico le permiten actuar con relativa independencia frente a las iniciativas colectivas de promoción del territorio.

Finalmente, es importante considerar su papel en el desarrollo socioeconómico de ambos territorios; es claro que ha motivado la consolidación de un mercado propio, específicamente en México, del que se ha valido para su posicionamiento empresarial y en el que otros empresarios han encontrado un medio para capitalizar oportunidades de negocio vinculadas a la oferta de servicios (hospedaje, alimentación, ocio, transporte...) y productos complementarios (quesos, artesanías, gastronomía regional...). 


\section{CONCLUSIONES}

En este artículo hemos analizado de manera multisituada el papel de una empresa trasnacional del mundo del vino en la promoción y en el desarrollo del enoturismo en dos territorios de Europa y América. A través del estudio de caso de Freixenet, hemos observado, por un lado, la reproduccción a escala (cf. Thomé et al., 2015) de un modelo de negocio global a través de alianzas internacionales y de la movilización de capital económico, político, social y cultural, construyendo y consolidando una marca-territorio usada para promocionar productos y servicios, así como para posicionarse como referente de la producción vitivinícola y del enoturismo en los territorios estudiados. Por otro lado, hemos podido observar también cómo determinados espacios rurales se insertan en procesos de globalización mediante prácticas trasnacionales asociadas a la reproducción de modelos de negocio; en nuestro caso, con un especial énfasis en el enoturismo.

La compañía Henkell-Freixenet, en su papel de empresa transnacional, nos sirve para contextualizar la especificidad, por una parte, de un modelo de producción instaurado, pero, por otra -y para nosotros más importante, si cabe- de un modelo de enoturismo desarrollado por la empresa en los territorios estudiados que en buena parte se reproduce a escala, de uno a otro territorio, y que da muestra de la mencionada inserción de espacios rurales marginales en procesos globales que insertan dichos territorios en un marco amplio de comunicación y de influencia económica y cultural.

El enoturismo, más allá de beneficios económicos concretos y cuantificables -que son claramente demostrables- sirve como una estrategia de asentamiento, de reafirmación de estatus y de reconocimiento de liderazgo en el sector vitivinícola. Sin embargo, a pesar de su importancia y de los amplios beneficios obtenidos directamente en la sede mexicana, el modelo enoturístico queretano de Freixenet no se adapta específicamente al territorio -más bien al contrario, es copiado directamente de los modelos europeos más estandarizados-, ni se cuida de manera particular, sino que se muestra como claramente secundario y subsidiario, favoreciendo las visitas masivas y coincidente con un modelo "clásico" de desarrollo turístico poco especializado y que apunta más a las grandes cifras que a las experiencias memorables.

Este hecho, sin embargo, no es -al menos, de momento- signo de fracaso. Más allá de los beneficios económicos, la empresa utiliza todos los recursos a su alcance para afianzar su imagen y su presencia en el territorio. Freixenet monopoliza la producción de vinos -en cantidad de producción y en ventas-, y lo mismo hace con las visitas turísticas, que redundan principalmente tanto en el afianzamiento de la imagen de marca como en la implantación territorial, cada día más indiscutible, de la empresa.

Las implicaciones posibles de este trabajo recaen principalmente en la gestión del enoturismo en los sectores público y privado. En el primero, para formular políticas públicas de desarrollo más inclusivas en las regiones vitivinícolas que han sido objeto, a través de subvenciones, de apoyos para proyectos relacionados con la valorización turística del vino y la gastronomía (rutas del vino, por ejemplo); en el segundo, para impulsar el liderazgo de las empresas vitivinícolas en el desarrollo de las áreas rurales tomando como eje transversal el enoturismo. Para otros empresarios relacionados directa o indirectamente (turoperadores, 
hoteleros, guías...) con el sector, la presencia de estas empresas puede servir para capitalizar nuevas oportunidades de negocio relacionadas con el desarrollo de productos turísticos complementarios de las actividades ofertadas en las bodegas.

La principal limitación de este trabajo se refiere a la imposibilidad de realizar generalizaciones a partir del estudio de un solo caso, aunque se hayan analizado dos unidades dentro del mismo. Además, recupera la perspectiva de un número reducido de actores, por lo que será importante ampliar en el futuro hacia otros cuyo ángulo de conocimiento sobre el mismo objeto de estudio contribuya a generar nuevas hipótesis. Futuros trabajos podrían indagar el impacto socioeconómico de las empresas multinacionales en la integración socioterritorial de las regiones vitivinícolas, así como su incidencia en la transformación de la ruralidad y su papel en la gobernanza de las rutas del vino.

\section{REFERENCIAS BIBLIOGRÁFICAS}

ACEVIN (2019). Informe de visitantes a bodegas y museos del vino asociados a las Rutas del Vino de España. Año 2018. Recuperado de https://www.wineroutesofspain.com/bd/ archivos/archivo967.pdf

Alberdi, J.C. (2018). El valor de la marca y la promoción turística. Claves del éxito de la ruta del vino de La Rioja Alavesa. Lurralde: Investigación y Espacio, 41, 5-32.

Almanza, L. (12 de mayo de 2017a). Querétaro, líder nacional en vinos espumosos. El Financiero. http://www.elfinanciero.com.mx/bajio/queretaro-lider-nacional-en-vinos-espumosos.html.

Almanza, L. (25 de mayo de 2017b). Freixenet espera a 12 mil visitantes en su Festival de la Paella. El Financiero. http://www.elfinanciero.com.mx/bajio/freixenet-espera-a-12mil-visitantes-en-su-festival-de-la-paella.html.

Almanza, L. (7 de agosto de 2017c). Freixenet recibió 15 mil visitantes en Vendimia de Querétaro. El Financiero. http://www.elfinanciero.com.mx/bajio/freixenet-estima-15mil-visitantes-en-vendimia-de-queretaro.

Appadurai, A. (2001). La modernidad desbordada. Dimensiones culturales de la globalización. Montevideo: Ediciones Trilce.

Ávila, A. (2015). Análisis del turismo alternativo en comunidades indígenas de Chiapas, México. Études caribéennes, 31-32. DOI : 10.4000/etudescaribeennes.7601.

Beck, U. (1998). ¿Qué es la globalización? Falacias del globalismo, respuestas a la globalización. Barcelona: Paidós.

Blanco, M. (2016). Freixenet: conquistando el mundo con un Cava de marca. En M. Alonso, M. Blanco y E. Furió (Coords.), El español: retos y oportunidades económicas y formativas en un contexto global (pp. 53-66). España: Septem Editores.

Brochado, A., Stoleriu, O. y Lupu, C. (2019). Wine tourism: a multisensory experience. Current Issues in Tourism, 1-19.

Calleja, P. (5 de octubre de 2018). El Tío Pepe se resiste a quedarse en casa. El País. https:// elpais.com/economia/2018/10/03/actualidad/1538561007_110309.html 
De Jesús, D. y Thomé, H. (2018). Patrimonio enogastronómico en el centro de México: estetización y autenticación como mecanismos de apropiación turística. Anthropology of Food, 13. Recuperado de https://journals.openedition.org/aof/8333

De Jesús, D., Thomé, H., Espinoza, A. y Vizcarra, I. (2019). Trayectoria territorial de la región enológica de Querétaro, México (1970-2017): enoturismo y calidad territorial. Cuadernos Geográficos, 58(2), 240-261.

De Jesús, D., Thomé, H. y Medina, F.X. (2020). Enoturismo y promoción del territorio. Análisis comparativo entre el nuevo y el viejo mundo del vino. PASOS. Revista de Turismo y Patrimonio Cultural, 18(3): 457-471.

Dwyer, L. (2015). Globalization of tourism: drivers and outcomes. Tourism Recreation Research, 40(3): 326-339.

Fávero, I, y Rotta, J. (2007). Enoturismo en la región uva y vino-Brasil. Estudios y Perspectivas en Turismo, 16(2), 133-146.

Ferreira, S. y Hunter, A. (2017). Wine tourism development in South Africa: a geographical analysis. Tourism Geographies, 1-23.

Furió, E. (2012). Freixenet: las burbujas centenarias. En M. Alonso y E. Furió (Coords.), Nuevo siglo, nuevos retos. Diez casos de estrategia empresarial (pp. 35-51). Asturias: Septem Editores.

Giménez, G. y Heau, C. (2014). El problema de la generalización en los estudios de caso. En C. Oehmichen (Ed.), La etnografía y el trabajo de campo en las ciencias sociales (pp. 347364). México: IIA-UNAM.

Guinjoan, E., Badia, A. y Tulla, A.F. (2016). El nuevo paradigma de desarrollo rural. Reflexión teórica y conceptualización a partir de la rural web. Boletín de la Asociación de Geógrafos Españoles, (71), 179-204.

Hernández, J. (2009). Tequila: centro mágico, pueblo tradicional. ¿Patrimonialización o privatización?. Andamios, 6(12), 41-67.

Holmes, M. R. (2017). Integrated rural wine tourism: a case study approach. Journal of Wine Research, 28(3), 216-238.

Jiménez, R.M. (2014). Globarruralización: cómo el medio rural se ve afectado por la globalización y las TIC. GeoGraphos, 5(67), 283-311.

Jociles, I.M. (2018). La observación participante en el estudio etnográfico de las prácticas sociales. Revista Colombiana de Antropología, 54(1), 121-150.

Lacarrieu, M. (2009). De "turistas" y "viajeros" en el mundo trasnacional: retos, desafíos y problemas del turismo cultural-patrimonial. Études caribéennes, 13-14. DOI: 10.4000/ etudescaribeennes.3792

Marcus, G.E. (2001). Etnografía en/del sistema mundo. El surgimiento de la etnografía multilocal. Alteridades, 11(22), 111-127.

Marín, G. y García, A. (2012). Introducción. En G. Marín, A. García y M. Daltabuit (Coords.), Turismo, globalización y sociedades locales en la Península de Yucatán, México (pp. 1-11). España: ACA-PASOS.

Medina, F. X. (2004) 'Tell me what you eat and you will tell me who you are'. Methodological notes on the interaction between researcher and informants in the anthropology of food. En J. McClancy, \& H. Macbeth (Eds.), Researching food habits. Methods and problems, (55-62). Oxford: Berghahn. 
Medina, F.X. (2011). Turismo del vino y desarrollo local en la región vitivinícola de TokajHegyalja (Hungría). En F.X. Medina, D. Serrano y J. Tresserras (Eds.), Turismo del vino. Análisis de casos internacionales (99-117). Barcelona: Editorial UOC.

Medina, F.X. (2015). Turismo y cultura en denominaciones de origen enogastronómicas: el caso de la región de Tokaj-Hegyalja (Hungría). International Journal of Scientific Management and Tourism, 3, 167-177.

Medina, F.X. y Tresserras, J. (Eds.). (2007). Patrimonio gastronómico y turismo cultural en el Mediterráneo. Barcelona: Universitat de Barcelona-IEMed.

Medina F.X. y Tresserras, J. (2008). Turismo enológico y rutas del vino en Cataluña. Análisis de casos: D.O. Penèdes, D.O. Priorat y D.O. Montsant. PASOS, 6(3), 493-509.

Millán, M.G. (2012). Las denominaciones de origen y las rutas del vino en España: un estudio de caso. Rotur, 5, 41-66.

Mitchell, R. y Hall, M. (2006). Wine Tourism Research: the State of Play. Tourism Review International 9(4), 307-332.

Molleví, G. y Fusté, F. (2016). El turismo gastronómico, rutas turísticas y productos locales: el caso del vino y el queso en Cataluña. Geographicalia, 68, 25-47.

Pedrero, Y. (2015). La estrategia de internacionalización: análisis comparativo de los mecanismos de entrada en mercados exteriores de seis empresas que operan en diferentes sectores de la economía. (Tesis de máster). Universidad de Barcelona.

Perret, G. (2011). Territorialidad y práctica antropológica: desafíos epistemológicos de una antropología multisituada/multilocal. KULA. Antropólogos del Atlántico Sur, 4, 52-60.

Peris-Ortis, M., Del Río, M. de C. y Rueda-Armengot, C. (Ed.) (2016). Wine and tourism. A strategic segment for sustainable economic development. Londres: Springer.

Rivero, P.J. (2017). La investigación multilocalizada en los estudios migratorios transnacionales. Aportes teóricos y prácticos. Trabajo y Sociedad, (28), 327-342.

Romero, A. y Vera, A.M. (2014). Las empresas trasnacionales y los países en desarrollo. TENDENCIAS, 15(2), 58-89.

Serrano, D. (2011). El turismo del vino: la lectura espacial de un binomio estratégico. En F.X. Medina, D. Serrano y J. Tresserras (Eds.), Turismo del vino. Análisis de casos internacionales (pp. 19-33). Barcelona: Editorial UOC.

Shaw, E. (1999). A guide to the qualitative research process: evidence from a small firm study. Qualitative Market Research: An International Journal, 2(2): 59-70.

Stake, R. (2000). Case studies. In N. Denzin y Y. Lincoln (Eds.), The SAGE Handbook of Qualitative Research (pp. 435-454). Londres: SAGE Publications.

Suremain (de), C. E. (2017). Cuando la alimentación se hace patrimonio. Rutas gastronómicas, globalización y desarrollo local (México). TRACE, 72, 165-181.

Thomé, H. Vizcarra I. y Espinoza A. (2015). Perfomancia y fractalización como herramientas de metabolización de los espacios rurales. El caso de la Ruta del Queso y el Vino de Querétaro. Spanish Journal of Rural Development, 6(1): 29-44.

Thomé, H. (2019). Una aproximación al turismo agroalimentario desde el enfoque SIAL. Evidencias del enoturismo en el centro de México. En M.C. Renard y J. Tolentino (Coord.), Red SIAL México. Diez años de contribución a los estudios de los Sistemas Agroalimentarios Localizados (pp. 69-86). México: RedSIAL-México/IICA/Yod Estudio. 
Tight, M. (2010). The curious case of case study: a viewpoint. International Journal of Social Research Methodology, 13(4), 329-339.

TomaVinoMexicano (octubre de 2019). Episodio 17. Finca Sala Vive By Freixenet [Audio en podcast] Recuperado de https://open.spotify.com/episode/1rQEzridsDEY0C68qJWjT0

TomaVinoMexicano (octubre de 2019). Episodio 18. Vinícola Vinaltura [Audio en podcast] Recuperado de https://open.spotify.com/episode/5L8rMshjKJqeCGzgtNWSVZ

Visentin, F. y Vallerani, F. (2018). A countryside to sip: Venice inland and the Prosseco's uneasy relationship with the wine tourism and rural exploitation. Sustainability, 10: 1-18.

Wang, C. (2020). Creating a wine heritage in Japan. Asian Anthropology, 1-16.

Yin, R. (2009). Case study research. Londres: SAGE Publications.

Zamora, J. y Barril, M.E. (2007). Turismo y vino. Un estudio formativo sobre la evolución de las rutas del vino en Chile. Estudios y Perspectivas en Turismo, 16(2), 171-193.

\section{CONTRIBUCIONES DE LOS AUTORES}

Daniel De Jesús Contreras: análisis, adquisición e interpretación de los datos; redacción y revisión crítica del contenido; aprobación final de la versión a publicar. F. Xavier Medina: concepción original del trabajo; análisis, adquisición e interpretación de los datos; redacción y revisión crítica del contenido; aprobación final de la versión a publicar.

\section{AGRADECIMIENTOS}

Al Consejo Nacional de Ciencia y Tecnología (CONACYT) de México por la beca mixta otorgada a Daniel para realizar una estancia de investigación en la Cátedra UNESCO de Alimentación, Cultura y Desarrollo de la Universitat Oberta de Catalunya (UOC) bajo la dirección del Dr. F. Xavier Medina. Y a esta última por las facilidades académicas, técnicas y de infraestructura para realizar la investigación en el marco de la línea sobre "Alimentación y Turismo", de la cual el Dr. De Jesús Contreras es investigador invitado permanente. 